\title{
CONTAMINACIÓN FÍSICO QUÍMICA EN ZONAS DE FRACKING
}

\author{
María Eugenia Guerrero Useda ${ }^{1}$.
}

${ }^{1}$ Ph.D. en Ciencias Físico Matemáticas de la Universidad Estatal de Moldova, Investigador asociado en CIDEA S.A.S., Bogotá, Colombia. mguerrerouseda@gmail.com

https://orcid.org/0000-0003-4428-1732.

\section{RESUMEN}

A pesar del consenso científico hacia la necesidad de limitar el calentamiento global, la urgencia por la provisión autónoma de recursos energéticos ha llevado a muchos Estados a autorizar proyectos que aplican técnicas no convencionales de extracción de combustibles fósiles, como es el caso de la perforación horizontal y la fracturación hidráulica de esquisto de gran volumen. Aunque son pocos los estudios que presentan evidencias concluyentes, estas técnicas son acusadas de acarrear peligros al ambiente y a la salud de las personas que trabajan y habitan zonas de fracking, de tal manera que los Estados están ante la disyuntiva de extender algunos años la autonomía energética exprimiendo hasta el final sus reversas de gas natural y petróleo, o buscar algún equilibrio con el planeta moviéndose hacia fuentes energéticas más sostenibles. A partir de la revisión de trabajos que presentan evidencias de contaminación física y química y otros impactos al ambiente en zonas donde se ha desarrollado la técnica del fracking, se presenta un panorama de riesgos para las personas que habitan cerca de plataformas de extracción y los peligros de desarrollar proyectos de fracking en zonas climáticas tropicales.

Palabras clave: análisis de peligros, fracturación hidráulica, perforación horizontal, riesgo.

Recibido: 21 de junio de 2021. Aceptado: 02 de agosto de 2021

Received: June 21, $2021 . \quad$ Accepted: August 02, 2021

DOI: https://doi.org/10.33571/rpolitec.v17n34a5

\section{PHYSICOCHEMICAL CONTAMINATION IN FRACKING AREAS}

\begin{abstract}
The urgency for the autonomous provision of energy resources has led to the application of unconventional techniques for extracting non-renewable resources, such as horizontal drilling and hydraulic fracturing of highvolume shale. Although there are few conclusive scientific studies, this technique is accused of bringing dangers to the environment and the health of people, in such a way that the states are facing the dilemma of extending some years of energy autonomy or one day seek balance with the planet. Based on the review of works that present evidence of physical and chemical contamination and other impacts to the environment in areas where the fracking technique has been developed, a pair of risks is presented for people who live near extraction platforms and the dangers to develop fracking projects in tropical climate zones.
\end{abstract}

Keywords: Hazard analysis, fracking, horizontal drilling, pollution.

Cómo citar este artículo: M.E. Guerrero Useda. "Contaminación físico química en zonas de fracking", Revista Politécnica, vol.17, no.34 pp.70-81, 2021. DOI: https://doi.org/10.33571/rpolitec.v17n34a5 


\section{INTRODUCCIÓN}

La fracturación hidráulica (fracking) junto a la perforación horizontal es una técnica estándar utilizada para acceder al gas y al petróleo que se encuentran atrapados en mantos rocosos de lutita, ampliamente aplicada en Estados Unidos donde se empezó a usar industrialmente desde el año 1949 y que ha generado grandes beneficios económicos. En los últimos años el fracking se ha extendido hacia otros países en donde se han estimado reservas técnicamente recuperables de gas, entre los cuales se incluyen países de la región latinoamericana como México, Venezuela, Colombia, Brasil, Paraguay y Argentina. Como actividad antrópica, esta técnica no está exenta de riesgos [1].

Varias publicaciones señalan que la aplicación del fracking tiene un alto potencia de causar contaminación ambiental; principalmente se le acusa de producir ruido [2], facilitar la emisión de la superficie de gases del subsuelo [3], de contaminar el aire [4] y de generar contaminación del agua y el suelo por lagunas de aguas residuales de extracción.

Con todo, solo a partir del año 2013 se empezaron a conocer algunos estudios científicos que reportan evidencias de contaminación en zonas donde se ha desarrollado la técnica [5], [6] y [7], sin que ello implique la presentación de modelos conceptuales de contaminación o evidencia de análisis de causa raíz.

La pregunta de investigación que guio la revisión fue la siguiente ¿Cuáles eventos de riesgo físico y químico relacionados con aplicación de la técnica del fracking y la perforación horizontal están documentados en la literatura científica reciente? Con la respuesta a esta pregunta se pretende dar luces sobre el posible impacto ambiental del fracking en zonas tropicales.

\section{METOdOLOGÍA}

Para el desarrollo de la revisión se aplicó la técnica documental con referencia en los trabajos [8], [9], [10] con aplicación de matrices bibliográficas y de análisis de contenido. Las nociones factor de riesgo, peligro, exposición, vulnerabilidad, incertidumbre, fracking, perforación horizontal y polución, estuvieron en el centro del análisis documental desarrollado sobre artículos publicados en revista científicas, informes de accidentes y alertas de riesgo de la industria de petróleo y gas.

Las nociones asociadas al análisis de riesgos se tomaron de [11], [12] y [13]. Del tesauro de la UNESCO se tomó la agrupación de términos cobijada bajo el número 2.60 del grupo ciencia <Polución, Catástrofe y Seguridad $>$ y de allí los términos principales: contaminación petrolera, contaminación de agua, contaminación atmosférica, contaminación sonora, contaminación radiactiva y calidad ambiental.

La revisión de tema se concentró en trabajos de la industria del gas y petróleo producidos principalmente en Estados Unidos; para el subtema de contaminación atmosférica, contaminación del agua y contaminación radiactiva por la industria petrolera también se revisaron trabajos de los países de la región latinoamericana principalmente Argentina [14], Brasil, Ecuador, México y Venezuela.

En el subtema contaminación sonora fueron revisados trabajos de fracking en zonas rurales y urbanas de Estados Unidos (Pensilvania), México [15] y Argentina (Formación Vaca Muerta), donde el suelo (árido y semiárido), el clima y los entornos ecosistémicos difieren significativamente de las zonas colombianas donde se han priorizado posibles pilotos para el fracking como el Valle Medio del Magdalena (Formación La Luna), con clima tropical. En total fueron revisados 32 artículos, 10 informes y 15 trabajos de literatura gris.

\section{FRACKING}


A continuación se listan algunos datos sobre la técnica, los que son necesarios para dimensionar la magnitud y alcance de la técnica de fracturamiento hidráulico, en adelante fracking. La técnica se utiliza para extraer petróleo y gas de bloques que están a una profundidad que oscila entre los 2500 y los 5000 metros; según la formación de roca, un pozo usado para fracking necesita entre $8000 \mathrm{~m}^{3}$ y $16000 \mathrm{~m}^{3}$ de agua y al menos 25 vagones de arena para preparar una mezcla de bombeado que típicamente se compone de agua (90\%), arena $(9.5 \%)$ y un coctel de productos químicos $(0.5 \%)$ [16]. Aunque recientemente la técnica ha variado en cuanto a la composición del líquido inyectado, sus componentes no se conocen en su totalidad [17] ya que no están en el dominio público por considerase secreto industrial. Se conoce que el líquido de reflujo y el producido se reutilizan parcialmente en la técnica de fracking [18].

Son muchos los trabajos de revisión que reportan la técnica de fractura hidráulica y los posibles peligros de polución del ambiente, especialmente de los recursos agua [19] y aire generados por fuentes de contaminación física y química [20], [21], [22], [16], [23], [24]; sin embargo, al analizar a profundidad los informes de incidentes reportados se encuentra que faltan datos de concentración de los contaminantes de interés antes y después de los incidentes, situación que suele ocurrir con frecuencia en la industria de petróleo y gas [23], [25].

De otra parte, al revisar la literatura tecno científica sobre el tema se encuentran estudios que se mueven en dos vías, por su cantidad en primer lugar estarían los que se enfocan en analizar el discurso político alrededor de fracking [4], las políticas públicas y la normativa relacionados con la extracción del gas de esquisto, estos trabajos son desarrollados principalmente en Estados Unidos y Reino Unido.

En un siguiente lugar, están los trabajos que revisan temas de seguridad y salud en el trabajo desarrollados principalmente en Estados Unidos, y que revisan accidentes en la industria del fracking y temas de salud ocupacional de los trabajadores de las plantas de exploración y extracción. Cabe señalar que para el 2009 ya se reportaba la existencia de más de 1.5 millones de millas de tuberías que conectaban pozos de extracción, plantas de procesamiento, instalaciones de distribución y distribución al cliente [3] y según datos de la Cámara de Comercio de Estados Unidos para el 2013 se contaba con 1.7 millones de empleos ya creados y un total de 3.5 millones proyectados para el 2035 para la industrial de fracking.

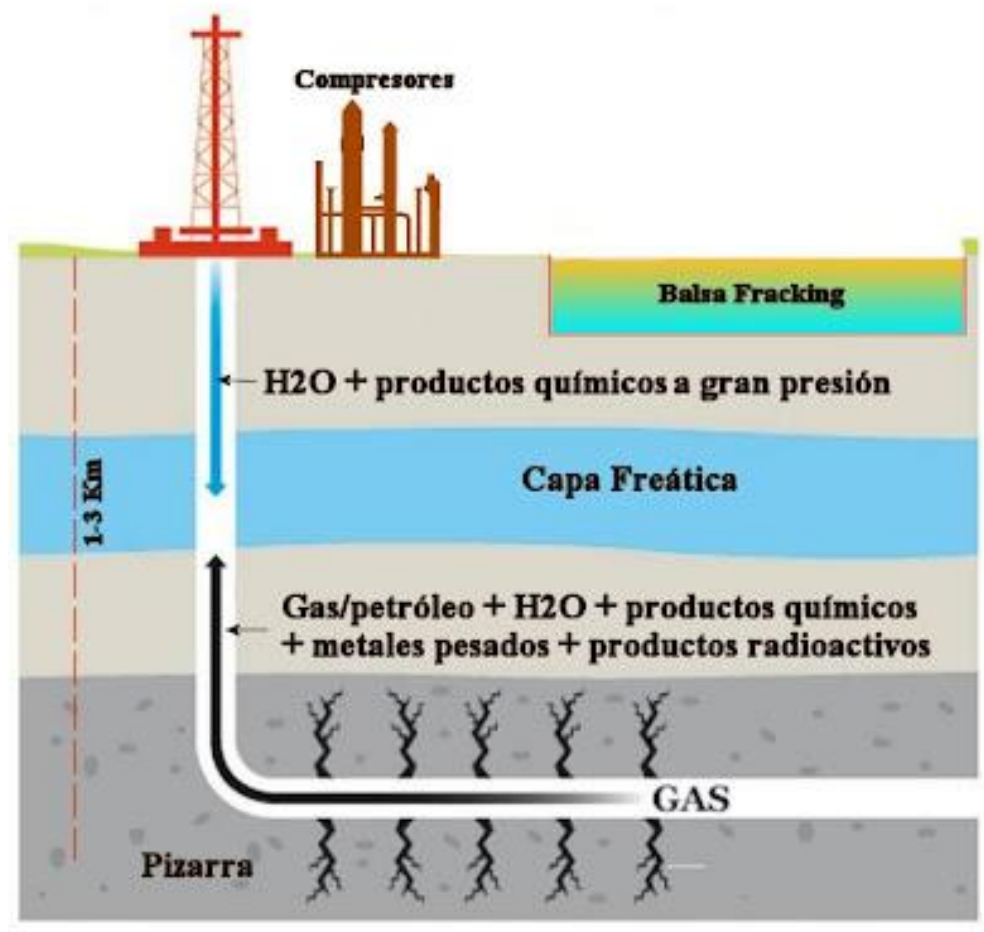


Fig. 1. Esquema general del proceso de fracturación hidráulica de un pozo. Fuente: Blog de Aula.

\section{RIESGOS POR EMISIÓN DE GASES Y DE POLVO DE SÍLICE}

Como se anotó, la fractura hidráulica se hace con el propósito de liberar gas y petróleo atrapado en el subsuelo. Además de gas de lutita, más conocido como gas de esquisto, a la superficie se liberan otros gases presentes en la roca generadora como el benceno, el metano, el radio y el radón, con lo cual se incrementa el riesgo de contaminación del aire sobre todo en las fases de construcción de plataformas y de perforación [21], [3].

Durante la última década se ha constatado el aumento rápido de metano en la atmósfera. Así, Robert W. Howarth concluye que la producción de gas de esquisto pudo contribuir con más de la mitad del aumento de las emisiones de los combustibles fósiles a nivel mundial y aproximadamente un tercio del aumento total de las emisiones de todas las fuentes [5]. Adicionalmente, Oyelakin reporta la emisión de valores tóxicos de metano en las zonas de almacenamiento de condensados de petróleo obtenidos en zonas de fracking [6].

Al fracking y a su red de instalaciones se asocian contaminantes primarios y secundarios con potencial para contaminar el aire. Además de metano, en [3] se reportan otros contaminantes de interés como el sulfuro de hidrógeno, vapores tóxicos de los componentes presentes en el fluido de fracturación hidráulica y contaminantes atmosféricos de criterio. Adicionalmente, Paulik y colaboradores [7] midieron mediante pulseras puestas en las manos de trabajadores y habitantes de zonas aledañas a pozos de extracción de gas natural y encontraron que son emitidos hidrocarburos aromáticos policíclicos (HAP) que contaminan el aire alrededor del pozo, aumentado la exposición personal trabajador y a los habitantes de la zona.

Entre 2016 y el 2018 en North Yorkshire, Inglaterra, fue monitoreada la calidad del aire y los parámetros meteorológicos (NOx, O3, NMHC, SO2, PM) en una zona rural llamada Kirby Misperton en la cual se desarrollaron actividades pre fracking; allí se estableció un incremento en tres veces de monóxido de nitrógeno y en dos veces del dióxido de nitrógeno (NO2), no se registraron cambios significativos en el material particulado en el aire o en los hidrocarburos no metanos. Las variaciones en la calidad del aire se atribuyeron al incremento de los vehículos y a la operación de equipos en el sitio [26].

\subsection{El metano}

Sobre el metano y el fracking se reportan varios trabajos que consideran un gran aporte en producción de gases efectos invernadero y la incidencia en el calentamiento global, estos trabajos en su mayoría aparecen publicados en revistas que no son de área de ingeniería ambiental y se refieren al metano producido por la combustión del gas o petróleo obtenido usando la técnica del fracking; sin embargo en [27] se estima que la huella de gases de efecto invernadero del gas de esquisto es mayor que la del carbón o el petróleo y que entre el 1.7 y el $6 \%$ del metano se ventila o se filtra a la atmósfera desde la cabeza del pozo, tuberías e instalaciones de almacenamiento.

Se ha establecido un aumento de emisiones de metano (hasta en un $2 \%$ ) en las zonas aledañas a pozos en donde se ha realizado fracturación hidráulica de Estados Unidos [28], este dato no es el mismo para otras zonas geográficas del fracking [29], sin embargo el debate se ha centrado frente al problema de la contaminación por metano del agua potable asociada con la extracción de gas de esquisto.

Así, Osborn y colaboradores [28] reportaron en 2011 altas concentraciones de metano en pozos de agua cercanos pozos con fracking en Pensilvania, este trabajo recibió varias críticas y dio pie para nuevos estudios que en 2013 concluyeron que el metano es común en los pozos de agua del condado de Susquehanna (Pensilvania) y su presencia se correlaciona mejor con la topografía y la geoquímica del agua subterránea, y menos con las actividades de extracción de gas de esquisto, por lo cual se concluye la falta de evidencia para atribuir la presencia del metano en pozos de agua al fracking desarrollado en la zona [30]. De otra parte, siguiendo a [31] se recomienda planificar y mantener una distancia prudencial entre las perforaciones de fracturación hidráulica y las fuentes de agua subterránea. 


\subsection{El radón $(\mathrm{Rn})$}

Es un gas radioactivo inerte, insípido, incoloro e inodoro; es el gas más denso conocido que se produce naturalmente por la descomposición del uranio. Se reportan al menos 34 isótopos de Rn que van desde Rn195 hasta Rn-228, de ellos son de interés tres: 222, 220 y 219, por depender del uranio. El primero es el más abundante en el ambiente (80\%). La vida media del radón alcanza 3.8 días, pero sus descendientes, el polonio 218 y el polonio 214, tienden a vincularse a partículas que al ser inhaladas pueden ser retenidas en diferentes tramos del aparato respiratorio. Los descendientes del radón tienen una vida media más corta y emiten radiación alfa (radiación ionizante, al igual que el radón 222) poco penetrante pero liberada a pocos micrómetros.

La inhalación de partículas sólidas contaminadas con descendientes de vida media corta del radón representa un alto riesgo para la salud, esto debido a que la radiación alfa puede impactar en las células del epitelio pulmonar, produciendo alteraciones moleculares, con probabilidad de causar cáncer de pulmón. Para Estados Unidos se ha estimado un nivel promedio de $1.3 \mathrm{pCi} / \mathrm{L}$ de radón en el aire interior, y de $0.4 \mathrm{pCi} / \mathrm{L}$ en el aire exterior, en este país se tiene como meta que el nivel de radón interior se aproxime al nivel exterior. En varias publicaciones se señala que con el incremento de operaciones de fracking en Estados Unidos, el nivel de radón en edificios ha aumentado y se invita al desarrollo de estudios detallados [32], [33], [34].

Como se indicó, el radón encontrado en el ambiente proviene de las actividades humanas. El radón pasa al ambiente a través del suelo principalmente por actividades en minas de uranio y fosfato, por fracking y por la combustión de carbón. Se estima que durante las operaciones de fracking es liberado gas radón al ambiente, sin embargo los estudios son pocos y no concluyentes. James Burkhart y colaboradores [32] desarrollaron mediciones durante diferentes fases del proceso de perforación y extracción de petróleo y gas en pozos ubicados en la cuenca de Denver (Colorado), donde se aplica la técnica de perforación horizontal y fracturación multihidráulica, en el sitio fueron constatados incrementos significativos de radón en las fases de la separación y de almacenamiento, donde el radón al aire libre fue de $4.5 \mathrm{pCi} / \mathrm{L}$, valor que está 10 veces por encima del nivel exterior según lo indicado en la normal EPA1992.

\subsection{BTEX}

El benceno, etilbenceno, tolueno y tres isómeros de xileno integran un grupo de contaminantes que se encuentra en los depósitos de petróleo crudo, carbón y gas. Este grupo es de interés reciente debido a que se ha reportado su presencia en pozos de agua potable de áreas de fracking [35]. Algunas empresas lo usan como compuesto para el fraccionamiento de fluidos, con el riesgo que en la fractura a través de la capa de confinamiento hidrológico pueda crearse una comunicación hidráulica entre la capa de carbón y de un acuífero subterráneo contaminándolo con BTEX.

Cabe señalar que no en todos los países las empresas están obligadas a comunicar los químicos que componen del líquido de fracturación, en algunos como en Australia, está prohibido el uso del BTEX en operaciones de fracturación [36], [29]. Ahora bien, la exposición a largo plazo a los componentes del BTEX afecta la salud ampliamente causando desde síntomas oculares y respiratorios, hasta afectaciones en la médula ósea y la sangre [37].

\subsection{Polvo de sílice}

La sílice está presente en varias de las operaciones de proceso de fracking. Las volquetas cargadas de arena de fracturación deben descargase en caliente en motores de arena, esta operación mecánica se constituye en una gran fuente de polvo de sílice (arena que contiene cuarzo) que es expulsado al medio circundante al que están expuestos los conductores de las volquetas de arena y los trabajadores del pozo. Los estudios [37] y [38] evidencian que la sílice cristalina constituye un riesgo potencial para los trabajadores.

\section{CONTAMINACIÓN SONORA POR FRACKING}


El fracking se relaciona con el incremento de la contaminación sonora y con el consecuente riesgo para la salud de las personas [39], [40] y para las especies de fauna que habitan en las zonas en las que se desarrolla esta actividad [41], sin embargo los estudios de ruido en las faenas de fracking son escasos.

Desde los procesos previos del fracking, los estudios sísmicos, la preparación, construcción, operación y desmonte de plataformas, los vehículos de abastecimiento de recursos y la operación de equipos, así como compresores y plantas generadoras, todos son fuentes de ruido que tienen un gran potencial para afectar la salud de los trabajadores, y reducir el confort de la fauna, como ya se ha mostrado en las aves migrantes.

La industria del fracking produce un complejo de ruidos transitorios y crónicos de diferentes fuentes, que están pendientes de ser estudiados a profundidad. Así, según lo reportado por Habicht, Hanson y Faeth en las plataformas durante el fraccionamiento el ruido llega a superar los 100 decibeles (dB) [42]; además durante más de dos meses se mantienen ruidos de aproximadamente 60-80 dB. En [2] se reporta la medición de ruido en las zonas de fracking en el suroeste de Pensilvania. En este estudio se constató que los niveles de sonido instantáneo durante el día oscilaron entre 45.0 y $61.0 \mathrm{dBA}$, mientras los dosímetros registraron niveles díanoche de 53.5-69.4 dBA en espacios abiertos y de 37.5-50.1 dBA en espacios cerrados.

Estos resultados coinciden con [40], que revisó un nivel de ruido promedio de 52 dBA con una desviación estándar de 10 para varios sitios en una plataforma de fracking. El nivel de ruido disminuye al alejarse del pozo (A menos de 90 metros se ha medido un máximo de $102 \mathrm{~dB}$, y a $2 \mathrm{~km}$ un máximo de $52 \mathrm{~dB}$ ) [43].

\section{DISCUSIÓN}

Actualmente, de la mayoría de pozos no convencionales en los que se implementa fracking se extrae gas de esquisto, que se estima es menos contaminante que el carbón y el petróleo. La tasa de recuperación de gas de un pozo oscila entre el 15\% y el $30 \%$ usando fracking, mientras la tasa de recuperación de gas convencional con pozos verticales llega a ser del $80 \%$.

Cabe señalar que con el advenimiento de la pandemia por el COVID-19 se redujo la demanda el petróleo a nivel mundial, esto afecto la industria de petróleo y gas; a la vez que aumentó la percepción de peligro en algunas comunidades frente a los riesgos ambientales y sobre todo frente a los riesgos biológicos emergentes. Incluso proyectos como Vaca Muerta en Argentina hoy están en tela de juicio: enfrentan mayores precios en la producción, menores precios en la venta, y la oposición de las comunidades mapuches. Alfonso López Suárez estima para Colombia una caída de al menos un $9 \%$ en la producción de crudo del país [44].

La revisión documental apunta a reconocer que las diferentes fases de la técnica del fracking conllevan riesgos latentes para los seres vivos. Los trabajadores de plataformas, los habitantes de las zonas aledañas a las plataformas y la fauna que habita los ecosistemas enfrentan peligros potenciales; de otra parte, los incidentes afectan principalmente a los trabajares (específicamente conductores y operadores que monitorean los pozos y las zonas de almacenamiento).

\subsection{Seguridad y salud de los trabajadores}

Para la industria del fracking el riesgo laboral, entendido como la posibilidad de que un trabajador sufra un determinado daño derivado del trabajo, es latente. En primer lugar, los agentes químicos del líquido de fractura no son conocidos en su totalidad por lo que su toxicología no es clara. De otra parte, aunque se conocen algunos informes de incidentes por explosiones debido a la acumulación de gases, son los accidentes automovilísticos con el $30 \%$ y los golpes con objetos con el $20 \%$ los que están en la cabeza de las causas de fatalidades en los trabajadores del sector [45].

Así, la CSB (Chemical Safety and Hazard Investigation Board) en un informe sobre una explosión de pozo de gas en Pryor Trust Well 1H-9U ocurrido en 2018 citó a [46] para señalar que para el periodo 2005-2009, esta industria presentó una tasa de mortalidad ocupacional 2.5 veces mayor que la industria de la construcción y 7 veces mayor que la industria general. 
En cuanto a los daños derivados del trabajo en fracking, concretamente las enfermedades profesionales se encontraron pocos estudios. En relación a la exposición al gas radón se encontraron trabajos que lo asocian con cáncer de pulmón [47] - [48] y trabajos que alertan sobre los impactos sobre la salud por el ruido crónico de los compresores [49].

\subsection{Vulnerabilidad de la fauna y la flora local}

Tanto el incremento de luz, ruido y polvo, como las aguas producidas por la fracturación hidráulica que contienen contaminantes químicos representan riesgos para la fauna. Lee y colaboradores [50] alertaron sobre el riesgo para las aves migratorias, lo que nos lleva a revisar posibles riesgos del fracking en zonas como el Valle Medio del Magdalena (Fig. 2) y el Distrito Especial de Barrancabermeja donde han sido asignados proyectos piloto para el fracking, específicamente en las zona donde confluyen las formaciones geológicas La Luna y Tablazo, en las cuales el potencial de recursos extraíbles es de gas y aceite a profundidades de 1300 a 5700 metros, con un espesor neto de 70-170 metros.

\section{POTENCIAL DE YNC EN COLOMBIA} CUENCAVALLEMEDIO DEL MAGDALENA

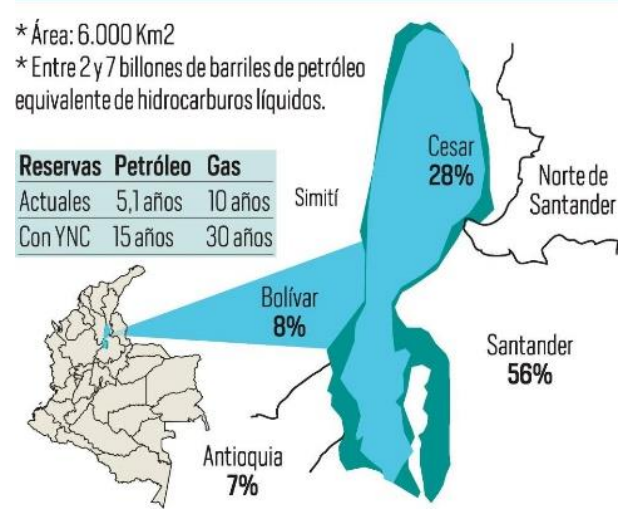

Fig. 2. Potencial de yacimientos no convencionales en la Cuenca del Valle Medio del Magdalena en Colombia.

La zona petrolera de Barrancabermeja alberga un rico sistema acuífero con varias ciénagas (El Llanito, Miramar, San Silvestre, Juan Esteban), lo que la convierte en un hábitat de gran biodiversidad de aves, mamíferos y peces. Pero debido a la confluencia de actividades antrópicas -la industria petroquímica, la agroindustria, el turismo, la caza y la pesca-, lo que sumado a la presencia de un relleno sanitario y al vertimiento ilegal de residuos de hidrocarburos que presionan la ciénaga de San Silvestre, varias especies «entre ellas iguanas, tortugas, nutrias, babillas, caimanes» se ven muy disminuidas.

Adicionalmente, especies únicas de la región como el mono araña café (Ateles hybridus) [52] y el manatí (Trichechus manatus) [53], ambas en peligro de extinción, están siendo afectadas. 


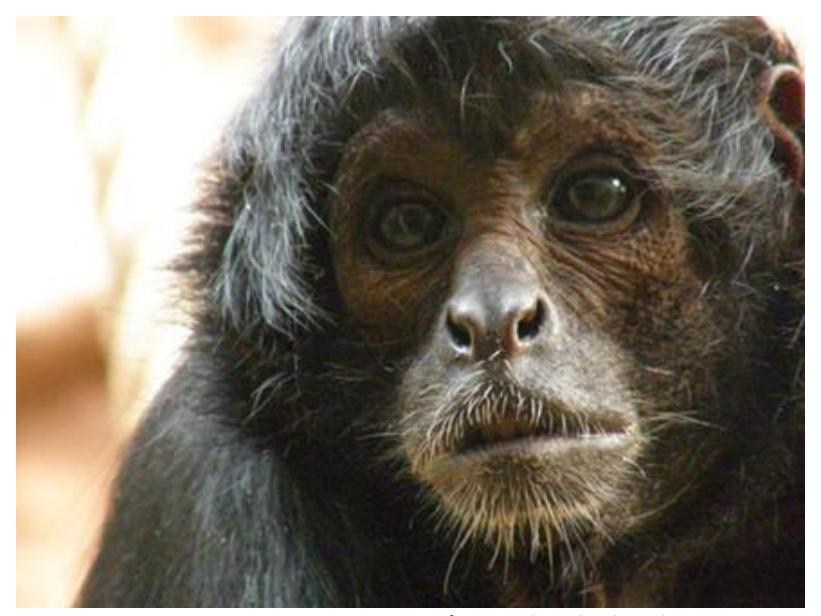

Fig. 3. Mono araña café (Ateles hybridus).

Cabe señalar que el ruido y la luz de las faenas de fracking perturban a las aves, muchas de las cuales son polinizadoras, lo que termina impactando la flora, ya afectada a consecuencia de derrames y afloramientos de petróleo (por ejemplo, las plantas o macrófitos acuáticos que participan en el ciclo del carbono de los ecosistemas acuáticos).

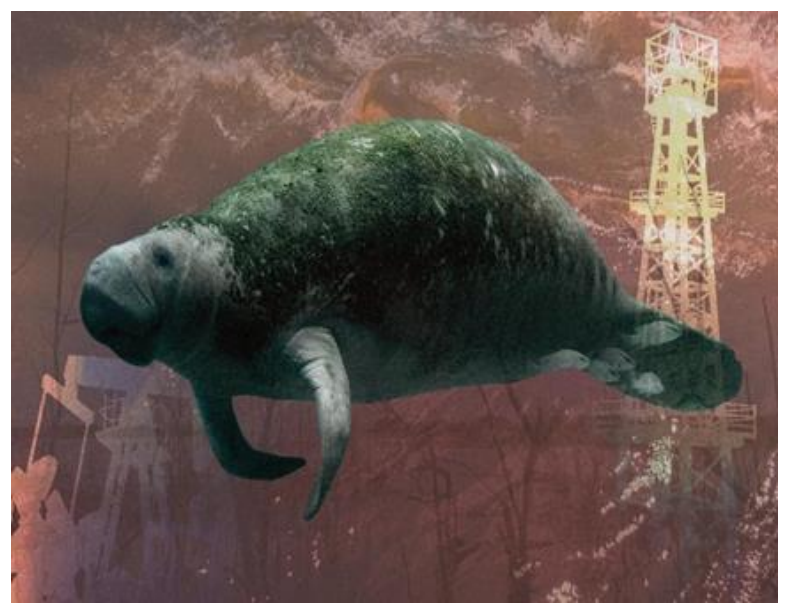

Fig. 4. Manatí (Trichechus manatus).

\section{CONCLUSIONES}

La contaminación del aire, las emisiones de gases «como el benceno, radón y compuestos tipo BTEX» y la contaminación sonora son hechos inevitables en las labores de perforación y fracking, por ello deben desarrollarse acciones de monitoreo continuo y sistemático a lo largo del ciclo de vida de los pozos en los que aplica esta técnica. En Colombia, las zonas priorizadas para proyectos piloto de fracking se encuentran en un piso altitudinal tropical y piso térmico cálido, zonas con gran riqueza ecosistémica, para protegerlas se requiere desarrollar estudios detallados -por operaciones y equipos- para determinar las variables que pueden afectar la salud de las personas y el confort animal, además de completar las líneas de base en materia ambiental ya señaladas por varios investigadores en los lineamientos para adelantar actividades PPII.

La mayoría de artículos publicados sobre impactos del fracking hacen referencia al peligro y al potencial de riesgo, muy pocos presentan evidencia de eventos de riesgo materializados por el desarrollo de esta actividad, como si lo hacen los informes de accidentes que son poco circulados o tienen una menor audiencia. La sílice 
cristalina, las partículas y el benceno son riesgos menos estudiados que requieren mayor atención. Los estudios sobre contaminación por radón en zonas de almacenamiento muestran la necesidad definir medidas de vigilancia y control, así como se hace necesario desarrollar análisis sobe posibles daños en salud para los trabajadores de las plataformas.

El ruido y la vibración en zonas de fracking afecta a las personas y a las especies faunísticas, este impacto debe ser considerado en los posibles proyectos que se adelanten en zonas de trópico. La geografía y la ecología de las cuencas del Valle Medio del Magdalena y del Cesar-Ranchería debe estar suficientemente descrita antes de proceder con proyectos piloto de fracking.

Se recomienda para el contexto local la vigilancia intensiva (control y medición de variables) y la transparencia en el reporte de información.

\section{REFERENCIAS BIBLIOGRÁFICAS}

[1] Campin, D. (2019). The Unknown Risks of Fracking. Asia Pacific Unconventional Resources Technology Conference, Brisbane.

[2] Richburg, C. M. y Slagley, J. (2019). Noise concerns of residents living in close proximity to hydraulic fracturing sites in Southwest Pennsylvania. Public Health Nursing, vol. 36, nº 1, pp. 310 .

[3] Weinhold, B. (2012). The Future of Fracking: New Rules Target Air Emissions for Cleaner Natural Gas Production. Environmental Health Perspectives, vol. 120, nº 7, pp. 272-279.

[4] Cotton, M., Imogen R. y James, V. A. (2014). Shale gas policy in the United Kingdom: An argumentative discourse analysis. Energy Policy, vol. 73, pp. 427-438.

[5] Howarth, R. W. (2019). Ideas and perspectives: is shale gas a major driver of recent increase in global atmospheric methane? Biogeosciences, vol. 16, p. 3033-3046.

[6] Oyelakin, O. A. (2016). Thermal-swing adsorption to capture and recover toxic vapor emissions from condensate storage tanks (Doctoral dissertation, Texas A\&M UniversityKingsville).

[7] Paulik, L., Hobbiea, K. A., Rohlman, D., Smith, B. W. y Scott, R. (2018). Environmental and individual PAH exposures near rural natural gas extraction. Environmental Pollution, vol. 241, pp. 397-405.

[8] Hoyos Botero, C. (2000). Un modelo para investigación documental, Medellín: Señal Editorial.

[9] Gómez Vargas, M., Galeano Higuita, C. y Jaramillo Muñoz, D. A. (2015). El estado del arte: una metodología de investigación. Revista Colombiana de Ciencias Sociales, vol. 6, nº 2, pp. 423 442.

[10] Guerrero Useda, M. E. (2015). Guia metodológica I. Revisión de tema, Bogotá.

[11] Enciso, L., Pacheco, D., Rivera D. y Guerrero Useda, M.E. (2014). Análisis de factores de riesgo en trabajadores de ladrilleras de Ubaté. IIEC, vol. 3, no 3, pp. 5-10.

[12] Bravo Mendoza O. y Sánchez Celís, M. (2012). Gestión integral de riesgos, 4 ed., vol. 1, Bogotá: B\&S.

[13] Porter, M. (2002). Natural hazard and risk managenent for south american pipelines. 4th International Pipeline Conference, Calgary.

[14] Rosa, L. y D'Odorico, P. (2019). The water-energy-food nexus of unconventional oil and gas extraction in the Vaca Muerta Play, Argentina, Journal of cleaner production, vol. 207, pp. 743750. 
[15] Sanchéz Cano, J. E., Barrios D. Á. y Pérez Domínguez, A. D. (2019). Viabilidad de usar fracking en la explotación de recursos no convencionales petroleros en áreas semiáridas de México. Desarrollo sostenible de zonas áridas y semiáridas frente al cambio climático, Durango: Universidad Juárez del Estado de Durango, pp. 74-92.

[16] Rosenman, K. D. (julio 2014). Hydraulic Fracturing and the Risk of Silicosi, Clinical Pulmonary Medicine, vol. 21, nº 4, pp. 167-172.

[17] Soeder, D. J. (2021). Impacts to Human Health and Ecosystems. Fracking and the Environment, Springer, pp. 135-153.

[18] Zanganeh, B., Soroush, M., Williams-Kovacs J. D. y Clarkson, C. R. (2015). Parameters Affecting Load Recovery and Oil Breakthrough Time after Hydraulic Fracturing in Tight Oil Wells. Society of Petroleum Engineers.

[19] Charry-Ocampo, S. y Perez, A. J. (2018). Efectos de la estimulación hidráulica (fracking) en el recurso hídrico: Implicaciones en el contexto colombiano. Ciencia e Ingenieria Neogranadina, vol. 28, no 1, pp. 135-164.

[20] Maloney, K., Young, J., Faulkner, S., Hailegiorgis, A., Slonecker T.y Milheim, L. E. (2018). A detailed risk assessment of shale gas development on headwater streams in the Pennsylvania portion of the Upper Susquehanna River Basin, U.S.A. Science of The Total Environment, vol. 610, pp. 154-166.

[21] Salinas Avellaneda, A. (2015). Criterios sanitario ambientales a tener en cuenta en los proyectos de "fracking". Revista de Salud Ambiental, vol. 15, $\mathrm{n}^{\circ}$ Especial XIII Congreso Español de Salud Ambiental, pp. 12-64.

[22] Arnedo Cárdenas A. E. y Yunes Cañate, K. M. (2015). Fracking: extracción de gas y petróleo no convencional, y su impacto ambienta. Cartagena.

[23] Sherilyn A. Gross, Heather J. Avens, Amber M. Banducci, Jennifer Sahmel, Julie M. Panko \& Brooke E. Tvermoes (2013). Analysis of BTEX groundwater concentrations from surface spills associated with hydraulic fracturing operations. Journal of the Air \& Waste Management Association, vol. 63, no 4, pp. 424-32.

[24] Vergel, M. y Becerra, L. (2020). Impactos del fracking y una mirada del panorama colombiano. Journal de Ciencia e Ingeniería, vol. 12, $\mathrm{n}^{\mathrm{o}}$ 1, pp. 264-274. https://doi.org/10.46571/JCI.2020.1.23

[25] Guerrero Useda, M. (2018). Ruptura de oleoductos por interferencia externa, daño ambiental y sostenibilidad en Colombia. Producción + Limpia, vol. 13, no 2, pp. 7-13. https://doi.org/10.22507/pml.v13n2a1

[26] Purvis, R. M., Lewis, A. C., Hopkin, J. R. Wilde, S. E. (2019). Effects of 'pre-fracking' operations on ambient air quality at a shale gas exploration site in rural North Yorkshire, England. Science of The Total Environment, vol. 673, pp. 445-454.

[27] Howarth, R. W., Ingraffea, A. y Engelder, T. (2011). Should fracking stop? Nature, vol. 477, $\mathrm{n}^{\mathrm{o}} 7364$, pp. 271-275.

[28] Osborn, S. G., Vengosh, A. N., Warner R. y Jackson, R. B. (2011). Methane contamination of drinking water accompanying gas-well drilling and hydraulic fracturing. Proceedings of the National Academy of Science, vol. 108, p. 8172-8176.

[29] Guo, M., Xu, Y. y Yongqin, D. C. (2014). Fracking and Pollution: Can China Rescue Its Environment In Time? Environmental Science \& Technology, vol. 48, no 2, pp. 891-892.

[30] Molofsky, L. J., Connor, J. A., Wylie, A. S., Wagner, T. y Farhat, S. K. (2013). Evaluation of Methane Sources in Groundwater in Northeastern Pennsylvania. Groundwater, vol. 51, n 3, pp. 333-349. 
[31] Moritz, A., Helie, J.F., Pinti, D. L., Larocque, M. y Barnetche, D. (2015). Methane Baseline Concentrations and Sources in Shallow Aquifers from the Shale Gas-Prone Region of the St. Lawrence Lowlands (Quebec, Canada). Environmental Science \& Technology, vol. 49, nº 7, pp. 4765-4771.

[32] Burkhart, J., Huber T., y Bolling, G. (2013). Potential Radon release during Fracking in Colorado. The American Association of Radon Scientists and Technologists. Spingfield, IL, 2027 de Radon Symposiun Pape, Spingfield.

[33] Carpenter, D. (2016). Hydraulic fracturing for natural gas: impact on health and environment. Reviews on Environmental Health, vol. 31, $\mathrm{n}^{\circ}$ 1, pp. 47-51.

[34] Bandreddy, N. A. (2019). Defining Correlation Between Radon, Uranium Deposits, and Oil and Gas Wells Using GIS Regression Methods. (Doctoral dissertation, University of Toledo).

[35] Leusch, F. y Bartkow, M. (2010). A short primer on benzene, toluene, ethylbenzene and xylenes. Griffith University.

[36] Queensland Government. (2018). Information sheet Petroleum and Gas. Fraccing and BTEX, 17 May.

[37] Srebotnjak, T. y Rotkin-Ellman, M. (diciembre 2014). Fracking Fumes: Air Pollution from Hydraulic Fracturing Threatens Public Health and Communities. NRDC Issue BRIEF, pp. 1-12. [38] Esswein, E. J., Breitenstein, M., Snawder, J., Kiefer, M. y Sieber, W. K. (2013). Occupational Exposures to Respirable Crystalline Silica During Hydraulic Fracturing. Journal of Occupational and Environmental Hygien, vol. 10, n 7.

[39] Hays, J., McCawley, M. y Shonkoff, S. (2017). Public health implications of environmental noise associated with unconventional oil and gas development» Science of The Total Environment, vol. 580, pp. 448-456.

[40] Assessing Environmental Impacts of Horizontal Gas Well Drilling Operations. (2013). Air, Noise, and Light Monitoring Results» Charleston.

[41] Todd, S. W., Hoffman, M. T., Henschel, J. R., Cardoso, A. W., Brooks, M., \& Underhill, L. G. (2016). The potential impacts of fracking on biodiversity of the Karoo Basin, South Africa. Hydraulic Fracturing in the Karoo: Critical Legal and Environmental Perspectives. Juta \& Company (Pty) Ltd., Cape Town, 278-301.

[42] Habicht, S., Hanson, L., \& Faeth, P. (2015). The potential environmental impact from fracking in the Delaware River basin. CNA Analysis and Solutions, Arlington, Virginia, USA.

[43] Werner, A. K., Vink, S., Watt, K., \& Jagals, P. (2015). Environmental health impacts of unconventional natural gas development: a review of the current strength of evidence. Science of the Total Environment, 505, 1127-1141.

[44] López Suárez, A. (10 mayo 2020). Precios y pandemia le quitarían reservas de petróleo a la Nación. PORTAFOLIO. https://www.portafolio.co/economia/precios-y-pandemia-le-quitarianreservas-de-petroleo-a-la-nacion-540681

[45] Goldstein, B. D., Brooks, B. W., Cohen, S. D., Gates, A. E., Honeycutt, M. E., Morris, J. B., ... \& Snawder, J. (2014). The role of toxicological science in meeting the challenges and opportunities of hydraulic fracturing. Toxicological Sciences, 139(2), 271-283.

[46] Witter, R. Z., Tenney, L., Clark, S., \& Newman, L. S. (2014). Occupational exposures in the oil and gas extraction industry: State of the science and research recommendations. American journal of industrial medicine, 57(7), 847-856.

[47] Resnikoff, M. (2011). Radon in natural gas from Marcellus Shale. Ethics in Biology, Engineering and Medicine: An International Journal, vol. 2, nº 1, pp. 317-331. 
[48] Tian, W., Wu, X., Liu, D., Knaup, A., Chen, C., \& Sondergeld, C. (2019). Investigating Effects of Pore Size Distribution and Pore Shape on Radon Production in Marcellus Shale Gas Formation. Energy \& Fuels, 33(2), 700-707. https://doi.org/10.1021/acs.energyfuels.8b03311 [49] Boyle, M. D., Soneja, S., Quirós-Alcalá, L., Dalemarre, L., Sapkota, A. R., Sangaramoorthy, T., \& Sapkota, A. (2017). A pilot study to assess residential noise exposure near natural gas compressor stations. PLoS One, 12(4), e0174310.

[50] Lee, A. T., Geary, C., Wright, D. R., \& Dean, W. R. J. (2019). Vulnerability of birds to contamina-ted water sources in the Karoo region of South Africa. Ostrich, 90(4), 397-406. https://doi.org/10.2989/00306525.2019.1638846

[51] Rodríguez Barajas, Y. C. (6 mayo 2019). Polémica porque el fracking en Colombia volvió a tomar fuerza. Vanguardia.

[52] De Luna Uribe, A. G. (2018). Ecología, densidades poblacionales y estado de conservación de los primates del Magdalena Medio colombiano con énfasis en uno de los primates más amenazados con la extinción en el mundo, el mono araña café (Ateles hybridus). (Tesis de la Universidad Complutense de Madrid).

[53] Solano Naranjo, D. (2020). Desarrollo de El Llanito: una construcción desde la comunidad, Bogotá. (Disertación doctoral, Universidad de los Andes). http://hdl.handle.net/1992/43738

[54] Guo, M., Xu, Y., \& Chen, Y. D. (2019). Environmental enforcement and compliance in Pennsylvania's Marcellus shale gas development. Resources, Conservation and Recycling, 144, 24-31. https://doi.org/10.1016/j.resconrec.2019.01.006

[55] Navarro, M. (30 abril 1994). El Magdalena medio: motor del país. El Tiempo. 